\title{
Nekrolog.
}

Alexis Hansky, Adjunkt-Astronom an der Nikolai-Hauptsternwarte zu Pulkowo, wurde am I 1. August d. J. in Ssimeis in der Krim von einem plötzlichen Tod ereilt.

Hansky wurde am 20. Juni 1870 geboren, besuchte das Gymnasium in Odessa, widmete sich nach Absolvierung der dortigen Universităt der Astronomie und stand seit 1895 in steter Verbindung mit der Pulkowoer Sternwarte, an der er 1905 zum Adjunkt-Astronomen ernannt wurde. Während seines mehrjăhrigen Aufenthalts in Meudon und Potsdam beschäftigte er sich eingehend mit astrophysikalischen und astrographischen Arbeiten, wobei ihn besonders das Studium der Bewegungen auf der Sonnenoberfläche und ihre Struktur interessierte. Dank seinen hervorragenden photographischen Kenntnissen gelang es ihm, wichtige Aufschlüsse uber die Bewegung der Granulationen zu gewinnen. Seine vielseitige astronomische Bildung machte ihn zu einem sehr schätzenswerten Teilnehmer an verschiedenen Expeditionen, welche astronomische Aufgaben verfolgten. Hansky beobachtete die Sonnenfinsternis im Jahre 1896 auf Nowaja Semlja und im Jahre 1905 in Spanien, worüber er teils im Bulletin der Akademie der Wissenschaften, teils in den Mitteilungen der Pulkowoer Sternwarte Bericht erstattet hat. Selbstverständlich war er Mitglied der von Pulkowo im Januar Igo7 nach Turkestan entsandten Expedition, die leider wegen ungunstiger Witterung keinen Erfolg hatte. Siebenmal bestieg er den Montblanc, um an dem Janssenschen Observatorium aktinometrische Beobachtungen und Schwerebestimmungen auszufuhren und den Versuch zu machen, die Korona unabhängig von Sonnenfinsternissen zu photographieren. Auf Spitzbergen hat er während der Russischen Gradmessung (1901) sehr wertvolle Pendelbeobachtungen zum Zweck der Schwerebestimmung angestellt.

Gerade in diesem Jahre ging der von ihm lange gehegte Wunsch, an einer Sonnenwarte in der Krim tätig zu sein, in Erfúllung. Beauftragt, das vom Hofstallmeister Maltzow der Pulkowoer Sternwarte geschenkte Observatorium in Ssimeis zu leiten, war er vor $2^{1 / 2}$ Monaten dorthin ubergesiedelt. Unter seiner Aufsicht war das Hauptinstrument bereits montiert und alles zur Ausfuhrung umfassender astrophotographischer und astrophysikalischer Beobachtungen Erforderliche fertiggestellt, als ein Herzschlag beim Baden seinem schaffensfreudigen Leben ein Ziel setzte.

Hansky verfügte uber eine ungewöhnliche Tatkraft und war der Wissenschaft mit Leib und Seele ergeben. Seine überaus sympathische Persönlichkeit erwarb ihm einen großen Freundeskreis, der ihn jetzt schmerzlich betrauert. Für die Wissenschaft und speziell für die Pulkowoer Sternwarte, deren Tätigkeit auf astrophysikalischem Gebiete gerade eben durch Besitznahme des neuen Observatoriums in der $\mathbf{K r i m}$ eine Erweiterung erfahren hat, bedeutet der Tod Hanskys einen schweren Verlust.

Pulkowo, 1908 Aug. 27.

O. Backlund.

\section{Observations of minor planet 1908 DT}

shown on photographs taken with 30 -in. Reflector, at the Royal Observatory, Greenwich.

The following are approximate positions of a faint planet discovered by $\mathrm{Mr}$. Melotte on photographs of Phoebe (Saturn IX) taken with the 30 -in. Reflector. The planet is shown as a short trail and appears to be about mag. 16.

Royal Observatory, Greenwich, 1908 Sept. 1.

\begin{tabular}{|c|c|c|c|}
\hline 1908 & $\begin{array}{l}\text { Gr. M.T. } \\
12^{\mathrm{h}} 20^{\mathrm{m}}\end{array}$ & $\begin{array}{c}\text { RA. app. } \\
0^{\mathrm{h}} 40^{\mathrm{m}} 17^{\mathrm{s}} \cdot 3\end{array}$ & $\begin{array}{l}\text { Decl. app. } \\
+0^{\circ} 43^{\prime} 53^{\prime \prime}\end{array}$ \\
\hline 2 & 1312 & - 38 & to $413^{6}$ \\
\hline 30 & 1259 & - $\quad 37 \quad 37.3$ & to 4033 \\
\hline
\end{tabular}

$P$. H. Cowell for Astronomer Royal.

(391) Ingeborg. Correzione all'effemeride (V. R. I. 35): 1908 Agosto $25+\mathrm{r}^{\mathrm{m}} 40^{\mathrm{s}}+3 \cdot 3$ Gr. I Im3. G. Zappa.

(544) Jotta. Korrektion der Ephemeride (V. R. I. 35): 1908 August $28+3^{\mathrm{m}} \mathrm{r} 9^{\mathrm{s}}+28:$. . F. Palisa.

Personalnotiz. Der Privatdozent für Astronomie und Geodäsie an der Universität zu St. Petersburg Dr. A. Iwanow wurde zum Professor der Astronomie an derselben Universität gewählt.

Anzeige betreffend Erneuerung des Abonnements siehe Nr. $427 \mathrm{I}$.

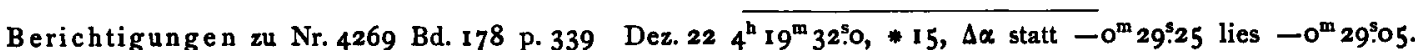

p. 343 Zum VI. Normalort ist zu bemerken, daß bei der Bildung des Wertes von $\Delta 8$ die erste Beobachtung von Dez. 22 ausgeschlossen ist wegen ungünstiger Sehnenlängen und $\mathrm{da} ß \mathrm{dem}$ so gebildeten Mittelwert der Beobachtung in Abo doppeltes Gewicht erteilt ist wegen der großen Zahl der Vergleichungen. So ergab sich der in den Normalgleichungen angewandte Wert $\Delta \delta=+0.3$.

Inhalt zu Nr. 4272. S. Chevalier, S. J. Occultation par Jupiter de l'etoile BD + $19^{\circ} 2095.385 .-$ E. E. Barnard. The Great Red Spot on Jupiter. 389. - E.Merlin. Sur la détermination systématique des éléments de la figure elliptique d'une planète au moyen de mesures micrométriques de diamètres. 391. - E. Hartwig. Utber den Algolstern 29.1907 (RZ) Aurigae und den Veränderlichen vom U Geminorum-Typus 31.1907 (SS) Aurigae. 393. - S. Enebo. Bestätigung der Veränderlichkeit einiger neu entdeckten Veränderlichen. 395. - A.A. Nijland. Das Juni-Minimum von RZ Ophiuchi. 397. - H. Kobold. Entdeckung eines neuen Kometen 1908 c. 397 - E. Hartwig. Var. 31.1907 Aurigae. 397. - O. Backlund. Anzeige des Todes von Alexis Hansky. 399. - P. H. Cowell. Observations of minor planet I908 DT. 399. - Kleine Mitteilungen. 399. 